\title{
Growth mechanism and characterization of zinc oxide microcages
}

\author{
Hong Jin Fan*, Roland Scholz, Florian M. Kolb, Margit Zacharias, Ulrich Gösele \\ Max-Planck-Institute of Microstructure Physics, Weinberg 2, 06120 Halle, Germany
}

Received 27 January 2004; received in revised form 10 February 2004; accepted 5 March 2004 by D.J. Lockwood

\begin{abstract}
We report the growth mechanism and structural properties of micrometer sized $\mathrm{ZnO}$ cages which were synthesized directly from $\mathrm{Zn}$ vapor deposition and oxidation. The $\mathrm{ZnO}$ microcages exhibit a hexagonal or spherical shape with partly or completely open surfaces and hollow interiors. The growth process of the microcages includes the deposition of $\mathrm{Zn}$ polyhedral particles, top face breaking of the $\mathrm{Zn}$ particles and $\mathrm{Zn}$ sublimation, and subsequent reaction to $\mathrm{ZnO}$. By controlling the various growth stages, we obtained information on the growth mechanism of the $\mathrm{ZnO}$ cages, which appears to be different from a mechanism reported previously. The chemical composition and crystalline structure were studied using energy dispersive X-ray spectroscopy and transmission electron microscopy, respectively. The room-temperature photoluminescence spectrum indicates a large quantity of oxygen-vacancy related defects within the wall of the $\mathrm{ZnO}$ cages.
\end{abstract}

(C) 2004 Elsevier Ltd. All rights reserved.

PACS: 81.10.Bk; 81.05.Dz; 68.65. - k

Keywords: A: Semiconductors; B. Crystal growth; C. Scanning and transmission electron microscopy

\section{Introduction}

Zinc oxide $(\mathrm{ZnO})$, a direct wide bandgap $(3.3 \mathrm{eV})$ semiconductor with a relatively high exciton binding energy $(60 \mathrm{meV})$, promises various applications in optical, electronic, and acoustic devices. In particular, $\mathrm{ZnO}$ microstructures have attracted increasing attention due to their UV lasing [1-3] and optoelectronic properties [4]. A number of nanometer and micrometer $\mathrm{ZnO}$ materials of varied geometries have been produced, e.g. nanowires, nanosprings, nanoneedles, nanowalls, coaxial cables, tubes, tetrapods and sheets [4-16].

Hollow $\mathrm{ZnO}$ structures are of particular interest. Due to their lower densities and higher surface areas compared to the bulk counterparts, hollow microstructures may find potential applications in filters, coatings, capsule agents for drug delivery, or templates for functional-architectured composite materials. Recently, Gao and Wang [17] reported on meso-porous $\mathrm{ZnO}$ cages/shells created from a mixture of

\footnotetext{
* Corresponding author. Tel.: +49-345-558-2760; fax: +49-345551-1223.

E-mail address: hjfan@mpi-halle.de (H.J. Fan).
}

$\mathrm{ZnO}, \mathrm{SnO}_{2}$, and graphite powder at high evaporation temperature $\left(>1000^{\circ} \mathrm{C}\right)$. A growth mechanism for the $\mathrm{ZnO}$ cages was proposed, which includes a process of solidification of liquid $\mathrm{Zn}$, oxidation, and $\mathrm{Zn}$ sublimation [17]. However, detailed information on the growth mechanism was rather limited in Ref. [17], partly because the different phases at different growth stages were experimentally not observed. In this letter, we report the successful synthesis of $\mathrm{ZnO}$ cages directly through thermal evaporation of metallic $\mathrm{Zn}$ powder and post-growth oxidation. We controlled the growth process in a way that the morphology and phases at different growth stages were obtained, which gives direct information on the growth mechanism. The growth mechanism of the $\mathrm{ZnO}$ cages concluded from our observations is quite different from the one proposed before [17].

\section{Experimental details}

Synthesis of $\mathrm{ZnO}$ cages was conducted inside a horizontal resistance furnace. A quartz or alumina boat 
loaded with metallic zinc powder (Aldrich, 99.998\%, 100 mesh) was transferred into the heating zone. Cleaned silicon pieces were located downstream at a position where the temperature was $\sim 300{ }^{\circ} \mathrm{C}$. The whole system was degassed by a turbomolecular pump to a background pressure of $\sim 10^{-5}$ mbar before starting the process with pure Ar. Thermal evaporation of $\mathrm{Zn}$ was realized at $800{ }^{\circ} \mathrm{C}$ under pure Ar flow $(20 \mathrm{sccm})$ using a chamber pressure of $200-$ $800 \mathrm{mbar}$. After $30 \mathrm{~min}$, an additional $\mathrm{O}_{2}$ flow $(5 \mathrm{sccm})$ was added to the initial $\mathrm{Ar}$ gas outside the furnace, and maintained for another $30 \mathrm{~min}$. After deposition the $\mathrm{O}_{2}$ flow was closed during the cooling process. The structure and composition of the synthesized products were characterized using a scanning electron microscope (SEM) equipped with an energy-dispersive X-ray spectrometer (EDS), and a transmission electron microscope (TEM). For TEM analysis, the sample was suspended ultrasonically in isopropanol and dispersed onto a TEM grid. Roomtemperature photoluminescence was excited by the $320 \mathrm{~nm}$ line of a $\mathrm{HeCd}$ laser and detected by a nitrogen cooled charge-coupled device camera (CCD).

\section{Results and discussion}

The successful synthesis of the desired products was found to be very sensitive to the $\mathrm{O}_{2}$ partial pressure in the reaction chamber. When the $\mathrm{O}_{2}$ flow was mixed to the $\mathrm{Ar}$ gas at the beginning of the heating process, most of the supplied $\mathrm{Zn}$ powder was oxidized near the source boat, where $\mathrm{ZnO}$ particles and/or tetrapod-like nanowires can be observed, while no obvious deposits were found at the end of the tube. Therefore, the addition of $\mathrm{O}_{2}$ gas flow only after the thermal evaporation process is a key factor for the formation of $\mathrm{ZnO}$ cages reported in our study. In addition to the cages, a large number of $\mathrm{Zn}$ polyhedral drum-like particles was also observed at a lower deposition temperature area $\left(<200^{\circ} \mathrm{C}\right)$. In this paper, we focus on the $\mathrm{ZnO}$ microcages.

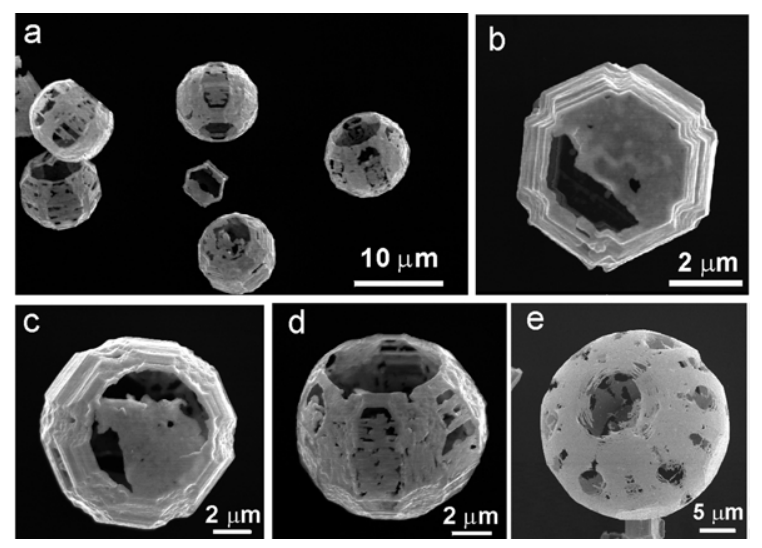

Fig. 1. SEM images of hollow $\mathrm{ZnO}$ cages.
The SEM images in Fig. 1 give a general overview of the collected products. The particles are hexagon-based or spherical cages, containing holes on their surface and an empty interior. The local wall thickness of the cages is not uniform, but varies with the crystallographic facets. The top (0001) surface is partly or completely open and the side faces can also be open in some cases, as seen in Fig. 1(d) and (e). Interestingly, for some cages, it can be clearly seen that the top face cracks and is ready to collapse downward. This implies that the top face breaks earlier, or energetically more easily, than the side faces during the growth. Moreover, it is noted that the surfaces of the $\mathrm{ZnO}$ cages are flat without being terminated by nanowires, in contrast to those in Ref. [17]. This is because no other catalytic metals are used in our experiments, whereas carbon-reduced $\mathrm{Sn}$ serves as a catalyst for the growth of $\mathrm{ZnO}$ nanowires via the vaporliquid-solid mechanism [18].

In order to elucidate the growth mechanism, $\mathrm{Zn}$ particles of different morphology, as possible earlier phases of the $\mathrm{ZnO}$ cages, were collected from different experiments or at different deposition positions. In Fig. 2(a), the Zn particles were collected at positions further away from the supply boat than the positioned Si substrate used for collecting the $\mathrm{ZnO}$ cages. The shape of the complete polyhedral particles implies that they are single crystals enclosed by (0001) top faces, (10-10) prismatic and other high-index faces $[19,20]$. Fig. 2(b) shows a representative $\mathrm{Zn}$ particle obtained at a position between where the $\mathrm{ZnO}$ cages and the $\mathrm{Zn}$ polyhedra
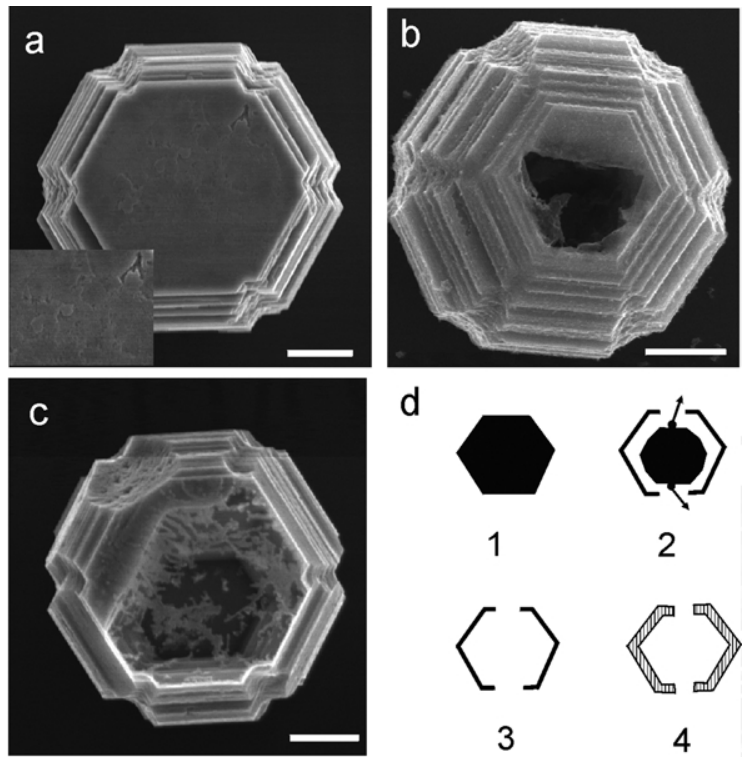

d
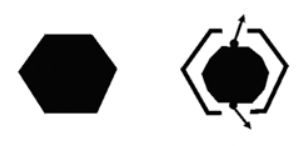

1

2
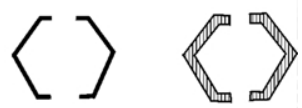

3

Fig. 2. SEM images of selected products arranged (a) - (c) in order to show the growth mechanism of the $\mathrm{ZnO}$ cages. The scaling bars are $1 \mu \mathrm{m}$. (a) A complete polyhedral $\mathrm{Zn}$ particle. Inset: enlarged view of part of the top surface. (b) A Zn particle whose top face is partly open. (c) A Zn particle with its widely open top surface and hollow interior. (d) Schematic of the formation process of the $\mathrm{ZnO}$ microcages. 
(see Fig. 2(a)) were obtained. Generally, they possess a hole on their top faces and partially hollow interiors. Fig. 2(c) shows a typical $\mathrm{Zn}$ cage obtained from additional experiments in which no extra $\mathrm{O}_{2}$ flow was introduced. These products, together with their respective growth conditions, are believed to represent the growth route of the $\mathrm{ZnO}$ cages, as discussed in more details below and illustrated in Fig. 2(d).

First, during the thermal evaporation and deposition process, $\mathrm{Zn}$ clusters aggregate into polyhedral crystals (as well as some other-shaped particles, see also Refs. [19,20]) induced by the supersaturation of $\mathrm{Zn}$ vapor at the low temperature region. The polyhedral crystals are deposited and distributed in a low density on the substrate (see Fig. 2(a) and (1) in Fig. 2(d)). A careful investigation of the $\mathrm{Zn}$ crystals shows that the top (0001) faces are not smooth but appear corrugated with some cracks. This could be an indication of crystal growth under the influence of a supersaturation gradient $[19,21]$. As a result of diffusion, the supersaturation near the edge of the growing crystal is higher than that at center, leading to a preferential nucleation and growth at the edges and corners. For the same reason, one could conclude that the atoms in the bulk of the $\mathrm{Zn}$ particle are 'weakly bonded' and the interior of the crystal posses structural imperfections. Such 'weak-bonding' will make the basal planes to be easily broken by oxidation (see below).

Second, at a temperature of $200-300{ }^{\circ} \mathrm{C}$, the residual oxygen and/or water vapor in the chamber oxidizes the $\mathrm{Zn}$ particle surface. The oxidation rate is different for the various crystallographic surfaces. The high energy basal faces are more easily oxidized than the low energy $\{10-10\}$, $\{10-11\}$ side faces. Moreover, due to the above-mentioned imperfections (cracks), the top $\{0001\}$ faces are also subjected to oxidation to a relatively high degree. For this reason and considering the short time $(\sim 30 \mathrm{~min})$, oxidation on the side of the polyhedral particles could be neglected compared to that of the basal faces. Therefore, the phase transformation-induced strain (i.e., volume expansion) causes the top $\{0001\}$ faces to break, as shown in Fig. 2(b) and (2) in Fig. 2(d). The breaking of the top faces occurs during the temperature increase and maintenance in pure Ar flow. Subsequently, the $\mathrm{Zn}$ inside the crystals can be sublimated $[11,17]$ by decomposition of the interior basal planes, and escape through the cracks in the top face. However, the edge remains intact in structure and $\mathrm{Zn}$-rich in composition. As a result, partly or completely hollow $\mathrm{Zn}$ particles are formed, as seen in Fig. 2(c) and assigned as (3) in Fig. 2(d). As a crucial condition a low oxygen partial pressure $\left(\sim 10-{ }^{5}\right.$ mbar in our experiment) is important. Higher partial pressure, in our experiments, results in wirelike structures terminating the side wall or extending from the interiors.

In the end, when extra $\mathrm{O}_{2}$ flow was introduced, the $\mathrm{Zn}$ hollow particles can be completely transformed into $\mathrm{ZnO}$ cages by oxidation of the walls (see Fig. 1 and (4) in Fig. 2(d)). Note that formation of holes on the side of the cages is also due to phase transformation induced strain.

On the basis of the above description, the initial $\mathrm{Zn}$ polyhedral single-crystal particles serve as templates for the eventual $\mathrm{ZnO}$ cages which grow in a 'casting' process. Gao and Wang [17] reported on mesoporous $\mathrm{ZnO}$ cages which are also supposed to originate from $\mathrm{Zn}$ polyhedral crystals. Briefly, it was assumed that liquid $\mathrm{Zn}$ droplets solidified into hexagonal or spherical $\mathrm{Zn}$ crystals, which were immediately oxidized by the residual $\mathrm{O}_{2}$ in the temperature region of $300-500{ }^{\circ} \mathrm{C}$. During the oxidation, nanometer sized $\mathrm{ZnO}$ islands were formed with nanogaps in between, as a result of the large $\mathrm{Zn}-\mathrm{ZnO}$ lattice mismatch. Subsequently, an increase in local temperature leads to the sublimation of $\mathrm{Zn}$ through the gaps, leaving a mesoporous $\mathrm{ZnO}$ cage structure. Although the reported growth process used in our study is similar, our results are not compatible with this model. As stated above, in our case, the $\mathrm{Zn}$ polyhedral particles were broken on their top $\{0001\}$ surfaces due to oxidation by residual oxygen or water vapor, whose partial pressure is lower than that in the former experiment [17]. Sublimation of $\mathrm{Zn}$ through the broken holes in the top $\{0001\}$ faces leads to formation of a Zn-rich cage structure. Only after the addition of a high concentration of oxygen a considerable phase transformation to $\mathrm{ZnO}$ occurs. This is in contrast to the case in Gao and Wang's study, where the formation of $\mathrm{Zn}$ particles and their oxidation occurred simultaneously [17].

The chemical composition of the hollow $\mathrm{Zn}$ particles and $\mathrm{ZnO}$ cages was verified by EDS. Fig. 3(a) shows the result from an individual hollow $\mathrm{Zn}$ particle corresponding to Fig. 2(c). The main composition is $\mathrm{Zn}$ while no oxygen peak was evident (the Si peak is from the substrate). Measurements on other forms of $\mathrm{Zn}$ particles in Fig. 2(a) and (b) give similar result. Fig. 3(b) and (c) show respectively the spot and scanning EDS profile recorded from an individual $\mathrm{ZnO}$ cage, which clearly exhibit $\mathrm{Zn}$ as well as $\mathrm{O}$ peaks. The $\mathrm{Zn}$ atomic percentage maximizes at the wall area of cages, and decreases abruptly in the center region of the scan line. These results agree well with the hollow structure of the examined particles. Due to the detection limit of the EDS for oxygen, it is not possible here to make an accurate quantitative interpretation of the atomic ratio between $\mathrm{Zn}$ and $\mathrm{O}$. Another indication of the phases of these products is that, upon annealing at $500{ }^{\circ} \mathrm{C}$ in air, particles in Fig. 2(a)-(c) can be transformed into $\mathrm{ZnO}$ clusters terminated with $\mathrm{ZnO}$ whiskers whereas those in Fig. 1 show no morphological change.

TEM was used to study the crystalline structure of the $\mathrm{ZnO}$ cages. Fig. 4 shows the TEM images of a fragment from the top and side face of a cage, and their corresponding electron diffraction (ED) patterns. The ED pattern for the top face piece can be indexed as a main [0001] (highintensity spots) orientation superimposed by others (lowintensity spots) in certain orientation relationship to each other. This suggests a [0001] texturing orientation of the top 

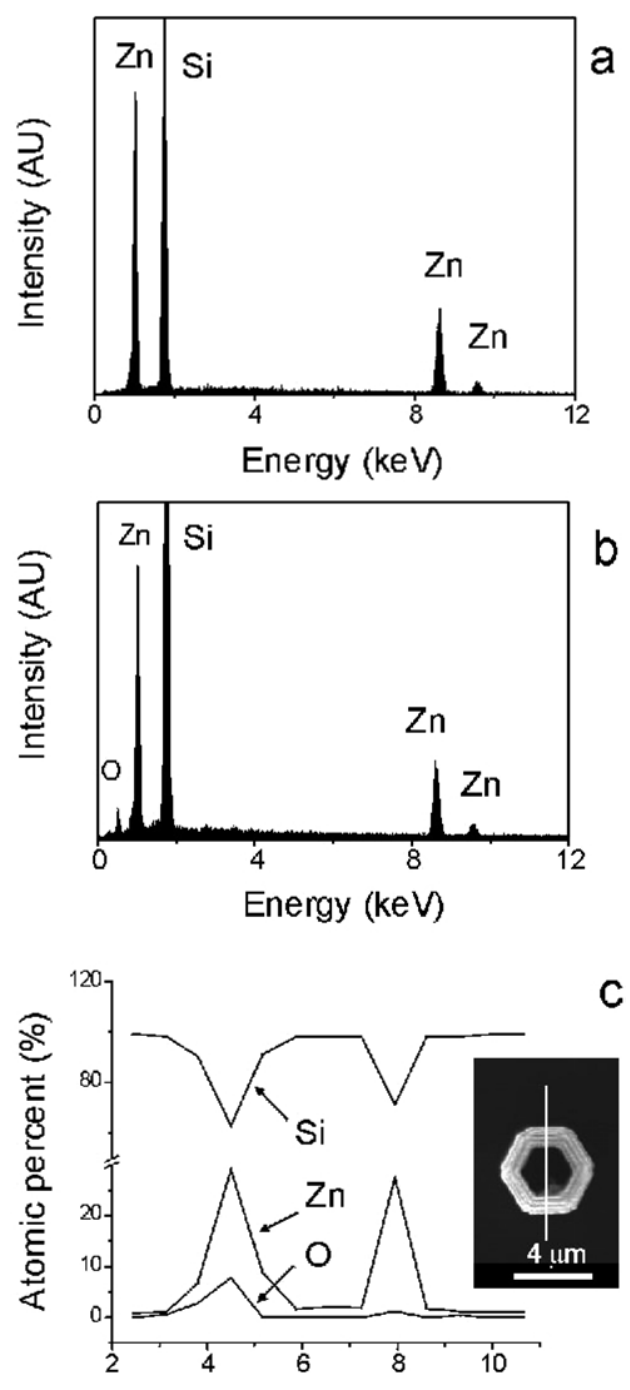

Relative position $(\mu \mathrm{m})$

Fig. 3. Representative EDS spectrum taken from (a) individual $\mathrm{Zn}$ hollow particles in Fig. 2(c) and (b) individual $\mathrm{ZnO}$ cages. (c) EDS line scan across a $\mathrm{ZnO}$ cage, confirming the existence of $\mathrm{O}$ and its hollow structure. The inset shows the SEM image with the direction of the line scan.

face of the cages. For the side piece the ED can be indexed as two sets: [10-10] and [0001], as marked in the pattern. Dark-field imaging verifies that the latter corresponds to the dark stripes in Fig. 4(b). Taking into account the structural correlation between the $\mathrm{ZnO}$ cages and polyhedral $\mathrm{Zn}$ single-crystal particles, the [10-10] set has its origin in the step $m$-face on the side, while the [0001] set is caused by the terrace $c$-face alternated by the step faces. The $c$-oriented terrace faces are bent somehow during the phase transformation from $\mathrm{Zn}$ to $\mathrm{ZnO}$, so that the (0001) ED pattern can be detected together with [10-10]. Taken together, our $\mathrm{ZnO}$ cages possess single-crystalline characteristics (i.e. texture)
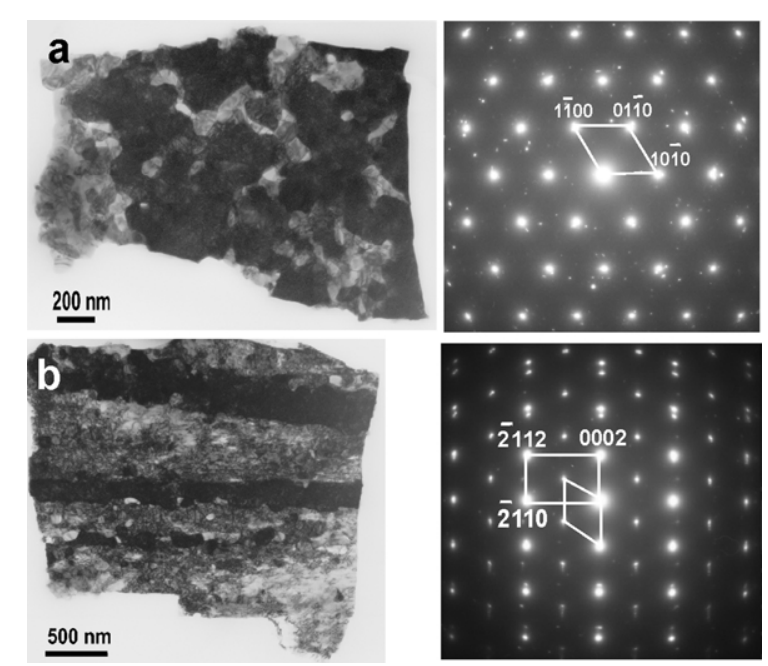

Fig. 4. TEM images of segments of the $\mathrm{ZnO}$ cages and their corresponding ED patterns. (a) Top face, (b) side face.

as revealed by the ED patterns. Such orientation texturing is consistent with that found in Ref. [17].

The light emitting properties of the $\mathrm{ZnO}$ cages were investigated by PL measurements. Fig. 5 shows the roomtemperature PL spectrum, which consists of a sharp peak at $378 \mathrm{~nm}$ and a broad peak centered at $\sim 534 \mathrm{~nm}$. The sharp peak corresponds to the near band edge emission of $\mathrm{ZnO}$, which results from the recombination of free excitons [22-24]. The broad and intensity strong deep level emission around $534 \mathrm{~nm}$ is most likely related to singly-ionized oxygen vacancies $[11,25]$. Therefore, the relatively strong green emission may indicate the existence of a high concentration of oxygen vacancies within the walls of the microcages.

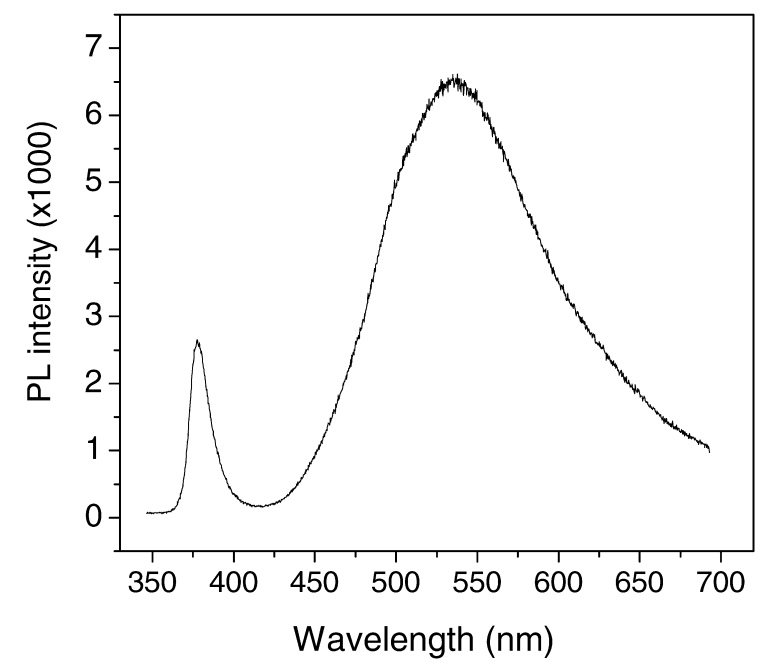

Fig. 5. Room-temperature photoluminescence spectrum of the $\mathrm{ZnO}$ cages. 


\section{Conclusions}

The synthesis of $\mathrm{ZnO}$ micrometer sized cages has been achieved directly through thermal evaporation of metallic $\mathrm{Zn}$ powder and post-oxidation. The controlled growth process allowed us to uncover the formation mechanism of the $\mathrm{ZnO}$ cages. It is demonstrated that the $\mathrm{ZnO}$ cages form via oxidation of as-formed hollow, or partially hollow, $\mathrm{Zn}$ particles. The latter become hollow as a result of $\mathrm{Zn}$ sublimation through large holes on the top $\{0001\}$ faces of the initial Zn crystals. Formation of such holes at the $\{0001\}$ faces appears to be due to phase transformation induced strain. The mechanical properties of the $\mathrm{ZnO}$ cages will be investigated in future.

\section{References}

[1] Z.K. Tang, G.K.L. Wang, P. Yu, M. Kawasaki, A. Ohtomo, H Koinuma, Y. Segawa, Appl. Phys. Lett. 72 (1998) 3270.

[2] Y. Wu, H. Kind, E. Weber, R. Russo, P. Yang, Science 292 (2001) 1897.

[3] J.H. Choy, E.S. Jang, J.H. Won, J.H. Chung, D.J. Jang, Y.W Kim, Adv. Mater. 15 (2003) 1911.

[4] H. Kind, H. Yan, M. Law, B. Messer, P. Yang, Adv. Mater. 14 (2002) 158.

[5] Z.W. Pan, Z.R. Dai, Z.L. Wang, Science 291 (2001) 1947.

[6] C. Liu, J.A. Zapien, Y. Yao, X. Meng, C.S. Lee, S. Fan, Y. Lifshitz, S.T. Lee, Adv. Mater. 15 (2003) 838.

[7] X.Y. Kong, Z.L. Wang, Nano Lett. 3 (2003) 1625.
[8] Y.W. Zhu, H.Z. Zhang, X.C. Sun, S.Q. Feng, J. Xu, Q. Zhao, B. Xiang, R.M. Wang, D.P. Yu, Appl. Phys. Lett. 83 (2003) 144.

[9] W.I. Park, G.-C. Yi, M. Kim, S.J. Pennycook, Adv. Mater. 14 (2002) 1841.

[10] H.T. Ng, J. Li, M.K. Smith, P. Nguyen, A. Cassell, J. Han, M. Meyyappan, Science 300 (2003) 1249

[11] J.Q. Hu, Q. Li, X.M. Meng, C.S. Lee, S.T. Lee, Chem. Mater. 15 (2003) 305.

[12] J.Q. Hu, Y. Bando, Appl. Phys. Lett. 82 (2003) 1401.

[13] L. Vayssieres, K. Keis, A. Hagfeldt, S.E. Lindquist, Chem. Mater. 13 (2001) 4395.

[14] V.A.L. Roy, A.B. Djurisic, W.K. Chan, J. Gao, H.F. Liu, C. Surya, Appl. Phys. Lett. 83 (2003) 141.

[15] H. Yan, R. He, J. Pham, P. Yang, Adv. Mater. 15 (2003) 402.

[16] J.Q. Hu, Y. Bando, H.J. Zhan, B.Y. Li, T. Sekiguchi, Appl. Phys. Lett. 83 (2003) 4414.

[17] P.X. Gao, Z.L. Wang, J. Am. Chem. Soc. 125 (2003) 11299.

[18] R.S. Wagner, W.C. Ellis, Appl. Phys. Lett. 4 (1964) 89.

[19] C. Nanev, D. Iwanov, J. Cryst. Growth 3/4 (1968) 530.

[20] S. Kasukabe, S. Yatsuya, R. Uyeda, J. Cryst. Growth 24/25 (1974) 315.

[21] Y.S. Park, D.C. Reynolds, J. Appl. Phys. 38 (1967) 756

[22] E.M. Wong, P.C. Searson, Appl. Phys. Lett. 74 (1999) 2939.

[23] Y.C. Kong, D.P. Yu, B. Zhang, W. Fang, S.Q. Feng, Appl. Phys. Lett. 78 (2001) 407.

[24] Y.G. Wang, S.P. Lau, H.W. Lee, S.F. Yu, B.K. Tay, X.H. Zhang, H.H. Hng, J. Appl. Phys. 94 (2003) 354.

[25] K. Vanheusden, W.L. Warren, C.H. Seager, D.R. Tallant, J.A. Voigt, B.E. Gnade, J. Appl. Phys. 79 (1996) 7983. 